\title{
Design of HIL Test System for VCU of Pure Electric Vehicle
}

\author{
Pengpeng $\mathrm{Nie}^{1, \text { a) }}$, Youyu $\mathrm{Wu}^{1, \text { b) }}$ and Xiaoyu Liang ${ }^{1, \mathrm{c})}$ \\ ${ }^{1}$ School of information, Wuhan University of Technology, Wuhan 430070, China. \\ a)niepengpeng2015@163.com \\ b)wuyouyul@whut.edu.cn.com \\ c)6237555@qq.com
}

\begin{abstract}
In order to achieve the test of function and strategy of pure electric car vehicle control unit(VCU) better, this paper designs a hardware-in-the-loop (HIL) test system for VCU based on NI-PXI platform. Firstly, expounding the principle of HIL testing technology and introducing the HIL test system plan based on demand analysis, then making sure the hardware which is used by system, and using it to build a vehicle model with MATLAB/Simulink successfully, finally running tests on the HIL testing system to demonstrate the political legitimacy and viability of the system.
\end{abstract}

Keywords: HIL testing system, VCU, design

\section{INTRODUCTION}

In order to improve the efficiency and reduce the cost, the automobile electronic system proposed the V-Mode development process, including function design and Simulink simulation, rapid prototyping development, automatic code generation of products, hardware-in-the-loop test and real vehicle calibration [1], so that every step of the development process can be verified [2]. VCU and some of the key components in HIL test are hardware-based while the others are model-based [3]. It not only overcomes the flaws of off-line simulation, but also overcomes the shortcomings of high cost and long period of real vehicle test [4]. However, most commercial HIL products are very expensive [5], which seriously hindered the promotion and marketization of HIL test technology.

Because of the fact that NI-PXI has the advantage of systems integration with software and hardware for HIL test [6], this paper designs a HIL test system for VCU of pure electric vehicle based on NI-PXI platform, which can test function and strategy of VCU with low cost and easy-using.

\section{SYSTEM DESIGN}

HIL test system can be divided into three levels, namely the signal level, power level and machine level [7]. And HIL test system is designed in this paper is a signal level test system, which is built based on the NI-PXI platform. In the test system, the vehicle model runs in real-time simulation environment, and the measurement signal is managed and controlled in the model. Besides that, VCU to be measured is in the form of hardware [8]. The specific features are integrated:

(1) Use external board through a specific response input and output to simulate the input and output of VCU.

(2) Use Veristand to develop the host computer interface, configure the hardware interface, and manage NI hardware resources.

(3) With the fault system, the physical channel of the board and the VCU to be tested are connected to realize the fault simulation function.

(4) The vehicle model built by Matlab/Simulink runs in the NI real-time system, in order to simulate the vehicle running environment and realize the hardware-in-loop-test. The specific system diagram is shown in figure 1. 


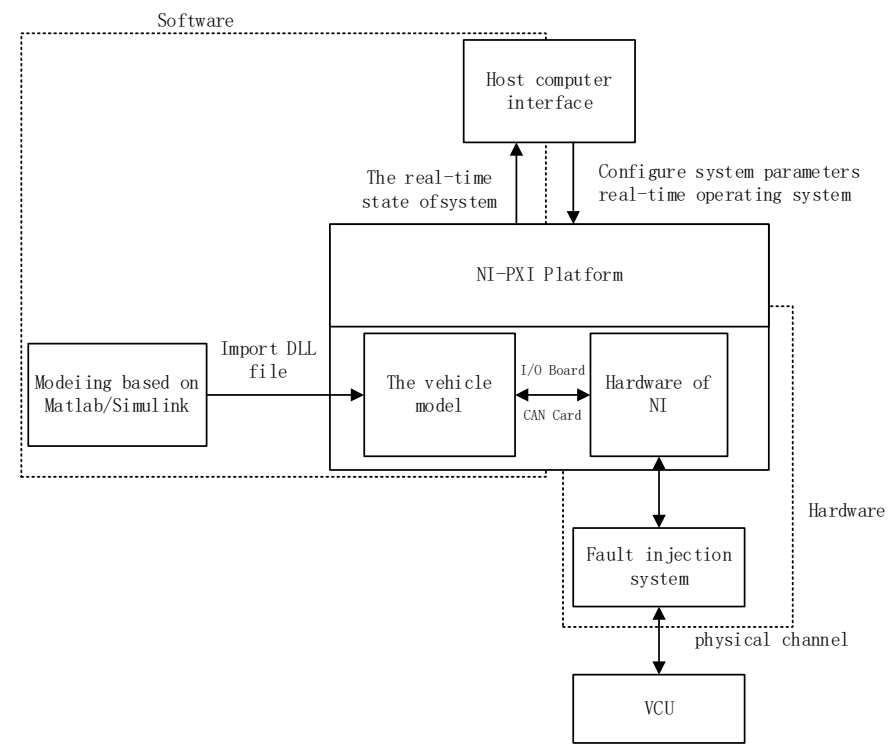

FIGURE 1. System solution

\section{HARDWARE DESIGN}

The hardware part of the HIL system is mainly based on the NI-PXI platform, Combined with the Fault Injection System and the connection wiring harness, it provides the input required for the real drive by the VCU, and collects the output response of the VCU's related pins in real time. The system is mainly composed of host, target computer, I / O board and CAN card, fault injection box and VCU to be measured. The specific structure is shown in figure 2.

NI-PXI platform provides hardware support for the system, including real-time processors and various types of boards and so on. According to the test requirements of the VCU, the selected hardware is shown in figure 3

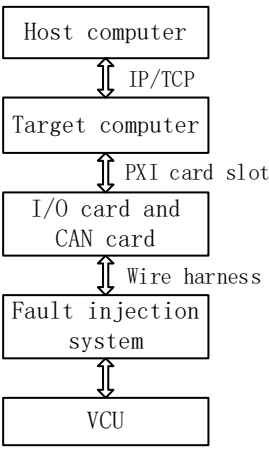

FIGURE 2. The hardware of system

\begin{tabular}{cccc}
\hline No. & Name & Description & Function \\
\hline 1 & PXIe-8840 RT & Real-time processor & Target computer \\
2 & PXI-8513 & CAN card & Simulation of vehicle CAN network \\
3 & PXI-6528 & Digital I/O card & \\
4 & PXI-6738 & Analog I/O card & Simulation of VCU's input signal \\
5 & PXI-2722 & Resistor card & Response to VCU's output signal \\
6 & PXI-6624 & PWM card & \\
7 & PXI-1078 & Chassis, 9 slot, 3U & Load real-time processor and cards \\
\hline
\end{tabular}

FIGURE 3. the hardware provide by NI

\section{SOFTWARE DESIGN}

The software pare of the HIL test system based on NI-PXI platform mainly includes the vehicle model built by MATLAB/Simulink and the host computer designed with Veristand.

\subsection{The design of vehicle model}

First of all, the vehicle model can be divided into several different model according to the different function, and the working mode of the pure electric vehicle mode on the basis of structure and working principle of vehicle is shown in figure 4 . 
(1) Battery Model .The inputs of this module are the demand power returned by the motor model and other parameters, and its outputs are actual output power, output voltage, working current and SOC (state of charge). The positive power represents discharge, and the negative power represents charge. The battery model is built on Thevenin model with first-order RC circuit, and the equivalent circuit can be seen from figure 5.

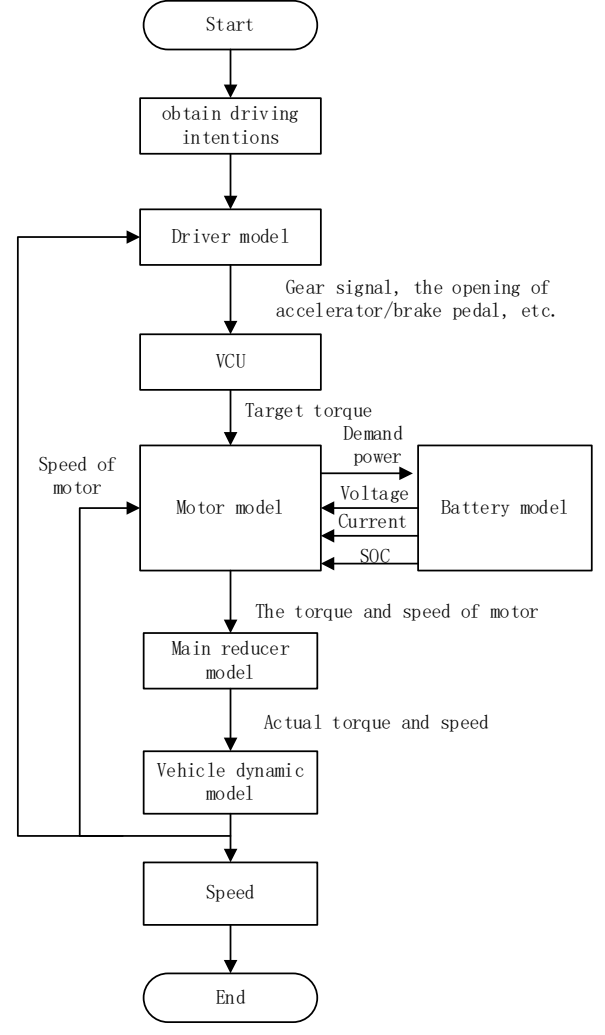

FIGURE 4 the working mode of vehicle model

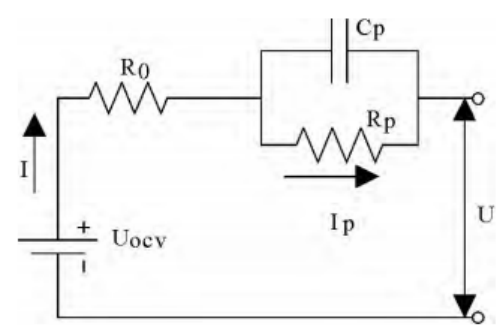

FIGURE 5. the equivalent circuit of the battery model The output voltage of the battery model is limited by the SOC and the maximum output power. When the value of SOC close to $100 \%$, the charging power is 0 , and when the value is close to 0 , the discharging power is 0 . In addition, the maximum output power is related to open circuit voltage $U_{\text {ocv }}$, the minimum operating voltage $\mathrm{U}_{\text {min }}$, and the internal resistance $\mathrm{R}_{\mathrm{int}}$, and the specific formula is shown in Equation(1).

$$
\mathrm{P}_{\max }=\frac{\left(\mathrm{U}_{\mathrm{ocv}}-\mathrm{U}_{\min }\right)^{2}}{\mathrm{R}_{\mathrm{int}}}
$$

SOC is the ratio of the residual capacity to the total capacity $\left(\mathrm{C}_{\max }\right)$. The value of SOC can be estimated by using ampere-hour method, and the basic idea is that the capacity of used this time $\left(\mathrm{C}_{\mathrm{used} 1}\right)$ can be obtained by integration of output current ( $\left.\mathrm{I}_{\mathrm{out}}\right)$ with time, the total used capacity $\left(\mathrm{C}_{\mathrm{used}}\right)$ is the sum of the value of $\mathrm{C}_{\mathrm{used} 1}$ and the capacity previously used. The formula is shown in Equation (2) and (3), and $\mathrm{SOC}_{0}$ represents the initial SOC.

$$
\begin{gathered}
\mathrm{C}_{\text {used }}=\int_{0}^{\mathrm{t}}\left(\mathrm{I}_{\text {out } / 3600}\right) \mathrm{dt}+\left(1-\mathrm{SOC}_{0}\right) \times \mathrm{C}_{\max } \\
\text { SOC }=\frac{\left(\mathrm{C}_{\max }-\mathrm{C}_{\mathrm{used}}\right)}{\mathrm{C}_{\max }} \cdot 100 \%
\end{gathered}
$$

(2) Motor model.The main inputs of the motor model include output voltage (U) and provided power from the battery model, and the model also receives the target torque sent by VCU through CAN and the actual speed (n) of motor from the vehicle dynamics model. Firstly, the maximum output torque of the motor can be obtained according to the current speed of the motor by table lookup, and then comparing the maximum output torque and the target torque, the value of motor's output torque $(\mathrm{T})$ is the minimum when the torque is positive or the maximum when the torque is negative. And we can look up the MAP figure to get the efficiency $(\eta)$ with the current speed and torque of the motor, other parameters such as the output power $\left(\mathrm{P}_{\text {out }}\right)$, the demand power $\left(\mathrm{P}_{\mathrm{dem}}\right)$ and output current can be calculated according to Equation (4) and (5).

$$
\mathrm{P}_{\text {out }}=\frac{\mathrm{T} \cdot \mathrm{n}}{9550}=\frac{\mathrm{T} \cdot \omega}{1000}=\mathrm{U} \cdot \mathrm{I}
$$




$$
\mathrm{P}_{\text {dem }}= \begin{cases}\mathrm{P}_{\text {out }} / \eta & \text { electric drive mode } \\ \mathrm{P}_{\text {out }} \cdot \eta & \text { electric brake mode }\end{cases}
$$

If the demand power is greater than the maximum working power of the motor, in order to protect the motor, it is necessary to add a power limit module to limit the demand power so that the working power of the motor will not exceed the maximum working power.

(3) Main reducer model. The function of this module is to reduce speed and increase torque, its inputs are the speed $\left(\mathrm{In}_{\text {speed }}\right)$ and torque ( $\left(\mathrm{In}_{\text {torque }}\right)$ from motor, and the module output the actual speed (Out $\left.\mathrm{Speed}_{\text {Spe }}\right)$ and torque (Out $\left.{ }_{\text {torque }}\right)$ after calculating with the transmission ratio $\left(\mathrm{T}_{\text {ratio }}\right)$ and transmission efficiency of the main reducer, and the specific method of calculation can be seen in Equation (6) and (7).

$$
\begin{gathered}
\text { Out }_{\text {Speed }}=\operatorname{In}_{\text {speed }} / \mathrm{T}_{\text {ratio }} \\
\text { Out }_{\text {torque }}=\left(\operatorname{In}_{\text {torque }}-\frac{\mathrm{d}\left(\text { Out }_{\text {speed }}\right)}{\mathrm{dt}} \cdot \mathrm{T}_{\text {intertia }}-\mathrm{T}_{\text {loss }}\right) \cdot \mathrm{T}_{\text {ratio }}
\end{gathered}
$$

(4) Vehicle dynamic model. The vehicle dynamic model can obtain the actual speed of the vehicle on the basis of the longitudinal dynamic equation, and then send the speed to the driver module to form a closed-loop test. According to the relevant knowledge of automobile theory, traction $\left(F_{t}\right)$ is the sum of rolling resistance ( $\left.F_{f}\right)$, air resistance $\left(F_{w}\right)$, slope resistance $\left(F_{i}\right)$ and acceleration resistance $\left(F_{j}\right)$, as shown in Equation (8). And the vehicle speed can be obtained by expanding this equation.

$$
\mathrm{F}_{\mathrm{t}}=\sum \mathrm{F}=\mathrm{F}_{\mathrm{f}}+\mathrm{F}_{\mathrm{w}}+\mathrm{F}_{\mathrm{i}}+\mathrm{F}_{\mathrm{j}}
$$

(5) Cycle condition model and driver model. The main function of the driver model in the manual test mode is to simulate the vehicle power logic and analyze the driving intention, including the analysis of the gear signal and accelerator / brake pedal signal, and then send the analytical physical signal to VCU. In the automatic test mode, introducing the PID controller to find the position of the accelerator / brake pedal according to comparing the different speed curves from the cycle condition model and the actual speed sent by the vehicle dynamic model, so that these two module can achieve the test of simulating different cycles conditions.

\subsection{Configuration of Veristand.}

Veristand is a data calibration software developed by National Instrument (NI) specifically for HIL test system. Figure 6 shows the functions of Veristand in the HIL test system designed by this paper.

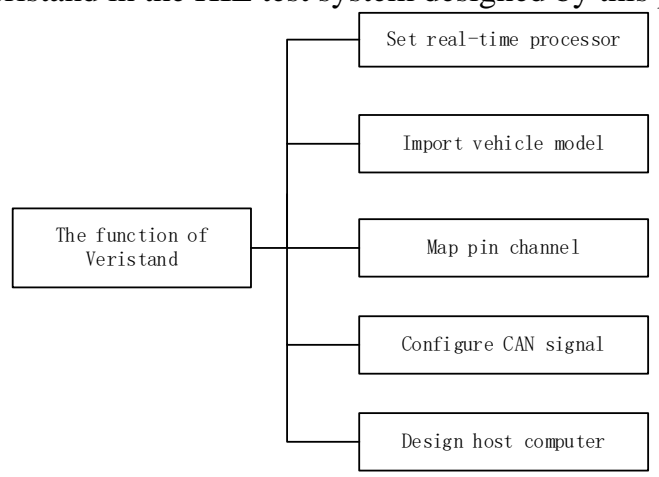

FIGURE 6. the function of Veristand

\subsection{Table of parameter}

The vehicle model of traditional HIL test system is customized for a certain vehicle, so the model needs to be modified first if the test object had changed, and the DLL file should be generate again, resulting in the workload of reuse the system becomes much larger. In order to solve the problem, the object-oriented programming idea is introduced creatively in the design of the model, and the approach practice is to design a table of parameter, which contains the detailed parameter information of the vehicle, motor and battery. The relevant information can be read and used from the table when the model runs, and this is a good way to avoid repeated and boring modifications to the model when it comes to experiments on different vehicles, the users just need to refill the table of parameter, and 
then the only thing needs to do is compile and run. Much time and human resources can be saved in this way. Besides, the risk of making mistakes when modifying the model will be reduced.

\section{ANALYSIS OF EXPERIMENTAL RESULTS}

The HIL test system can simulate the vehicle logic power and the process of acceleration and deceleration. And the test result can be seen in figure 7 and figure 8. The fault test mainly simulates the working state of VCU in the process of vehicle simulation, and the test of cycle condition is mainly to test the driving range and energy consumption. Users can determine whether the VCU meets the requirements based on the test results.

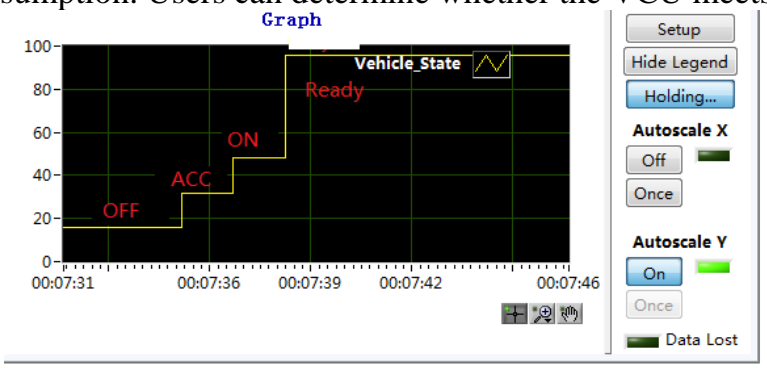

FIGURE 7 the state of the vehicle

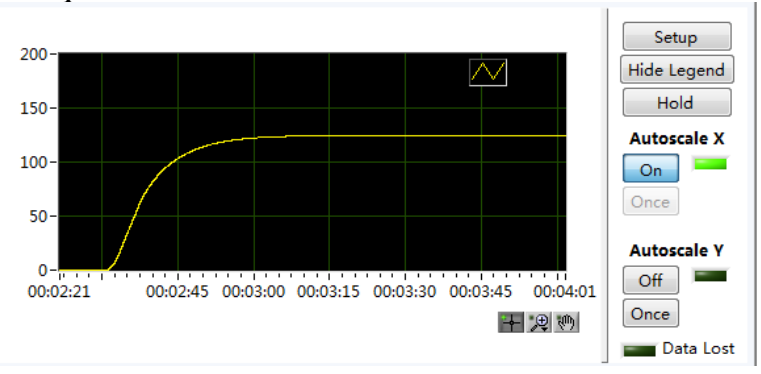

FIGURE 8. the speed change curve of the vehicle

\section{CONCLUSION}

Based on the analysis of the basic principle and composed meaning of HIL test system, this paper designed a HIL test system based on Veristand with the mainframe and tablet provided by NI-PXI platform integration, coupled with fault injection in system and other accessories, and by using MATLAB/Simulink to build a vehicle model for the configuration of the vehicle parameters to simulate the vehicle running environment, realize the hardware in the loop test to test VCU. The experiment shows that the system has successfully simulated the whole process of power supply, the process of acceleration and deceleration, the fault detection and the simulation of the working conditions. There is no obvious differences during experimental data and design goals. As a result, the system can test the design of VCU and provide basis data to the next vehicle test. Costs are far below traditional business HIL test system, and there will be good prospects for the new energy vehicle market.

\section{REFERENCES}

1. Zhang Heng, Research of the Hardware in the loop Simulation System for VCU of the Electric Vehicle, Jilin University, Changchun, 2013.

2. Tian Zhen, Zhang Xueman, Dong Tingting, et al, Development of a Vehicle Control System and Model Unit Test Based on V-mode, Chinese Journal of Automotive Engineering, 2(2012)458-463.

3. Wang Hongyu, Yuwen Zhiqiang, Fang Yong, QiaoYunqian, Development of pure electric vehicle powertrain controller based on hardware in the loop platform, 2015 6th IEEE International Conference on Software Engineering and Service Science (ICSESS), (2015)498-502.

4. Jin Xiaofei, Zhao Han, Li Yang, Development of Vehicle Control Unit Based on dSAPCE Hardware Simulation for Electrical Vehicle, Mechanical \& Electrical Engineering Technology, 8(2014)1-6.

5. Jinglin Xia, Qinghua Diao, Wei Sun, et al, Development of low cost hardware-in-the-loop test system and case study for electric vehicle controller, 2016 International Conference on Applied System Innovation (ICASI), (2016)1-4.

6. Ni Jimin, Li Xiaojin, Shi Xiuyong, Design of Host Progrom for Engine ECU HIL System Based on NI PXI Platform, Automobile Technology, 3(2014) 54-58.

7. A. Bouscayrol, Different types of Hardware-In-the-Loop simulation for electric drivers, 2008 IEEE International Symposium on Industrial Electronics, (2008)2146-2151.

8. Thanh Vo-Duy, Minh C. Ta, A signal hardware-in-the-loop model for electric vehicles, ROBOMECH Journal, 3(2016):1-11. 